Ann. Zootech., I968, 17 (4), 459-466.

\title{
ESSAIS D'ESTIMATION DE L'ÉPAISSEUR ET DE L'IMPORTANCE DE LA PEAU CHEZ LE POULET
}

\author{
F.-H. RICARD \\ avec la collaboration technique de G. MArche \\ Station expérimentale d'Aviculture du Magneraud, 17 - Saint-Pierre d'Amilly \\ Institut national de la Recherche agronomique
}

SOMMAIRE

Sur un lot de 25 poulets, nous avons mesuré l'épaisseur de la peau au niveau de l'aile, au niveau d'un pli cutané reliant la poitrine et la cuisse, ainsi qu'au niveau de la patte. Nous avons disséqué et pesé l'ensemble de la peau de la carcasse ainsi que la peau des ailes, des cuisses et pilons, et des pattes.

Le poids total de peau de la carcasse est fortement lié au poids de cette carcasse $(r=0,8)$ mais relativement peu à l'état d'engraissement $(r=0,4)$.

Les mesures d'épaisseur sont peu précises, à l'exception de la mesure directe faite au niveau de la patte. Leur intérêt, comme estimation de la finesse intrinsèque de la peau, reste à préciser par une expérimentation plus complète. I.es corrélations entre les épaisseurs et le poids total de peau ne sont pas significatives, à l'exception de l'épaisseur mesurée au niveau de l'aile.

Parmi toutes les variables étudiées, le poids de peau du groupe cuisse + pilon constitue la meilleure estimation du poids total de la peau chez le poulet.

\section{INTRODUC'TION}

La peau du poulet intervient dans la présentation de la carcasse ef c'est en même temps un élément consommable. Outre sa couleur, qui divise les consommateurs selon les régions, la peau peut se caractériser par son importance, sa finesse, sa texture, son grain. Si on veut améliorer chacune de ces caractéristiques, il faut pouvoir les mesurer, puis étudier les facteurs de leur variation.

Peu de travaux sont consacrés exclusivement aux caractéristiques de la peau du poulet. Il est possible de trouver quelques indications dans des études plus générales sur la qualité de la carcasse et il s'agit le plus souvent de données sur le poids de la peau. Ce poids de peau comprend toujours de la graisse sous-cutanée, si bien qu'il est lié non seulement au poids de la carcasse (voir par exemple LATIMER, I924, ou MCNAI,LY et KRAFT, I958) mais aussi à l'état d'engraissement du poulet (MCNAL, ${ }_{4}$ Y et KRAFT, I958; EsSARY et al., I96I). 
Quelques auteurs ont mesuré 1'épaisseur de la peau : DOI.ECEK et al. (I94I) prennent l'épaisseur d'un pli cutané au niveau des ptérylies du cou et de la poitrine en vue d'estimer indirectement l'état d'engraissement; MCNAII,Y et KRAFT (I958) veulent voir s'il existe des différences entre souches; ADAMs et al. (I967) étudient l'épaisseur de la peau qui recouvre le bréchet en tant que mesure indirecte de l'aptitude à la formation d'ampoules au bréchet chez le dindon.

Dans le présent travail, nous nous sommes intéressés à l'épaisseur et au poids de la peau. Nous étudions plusieurs variables susceptibles d'estimer ces caractères, si possible de façon indépendante du poids du poulet et de son état d'engraissement.

\section{MATÉRIEL, E'T MÉTHODES}

Nous avons travaillé sur 25 coquelets de la souche expérimentale Bresse-pile, abattus à l'âge de 88 jours. Quelques-uns des résultats obtenus ont été vérifiés sur d'autres poulets issus de la même souche.

Nous avons pesé la carcasse éviscérée, le dépôt gras abdominal (graisse située autour du gésier et contre la paroi abdominale, sans y ajouter la graisse intestinale) et les deux pattes, sectionnées à l'articulation pilon-patte. Je poids de la carcasse éviscérée représente ici le poids global du poulet. Dans un travail précédent (DELPECII et RICARD, I 965), nous avons montré que le dépôt gras abdominal était un bon indice de l'état d'engraissement du poulet. Mais il s'agit d'une variable qui ne suit pas une distribution normale et nous avons été amenés à utiliser la racine carrée du poids de cette graisse abdominale, comme nous l'avions fait précédemment (RICARD et RouviER, 1967).

\section{Description des mesures prises}

Nous avons étudié quatre mesures d'épaisseur, répétées trois fois à droite et trois fois à gauche, prises en des endroits où il y avait aussi peu que possible de graisse sous-cutanée :

I. Épaisseur de la membrane de l'aile, c'est-ì-dire de la peau comprise entre les deux premiers segments de l'aile, à proximité de l'articulation bras-avant-bras.

2. Épaisseur d'un pli cutané obtenu en pinçant la peau comprise entre la cuisse et la poitrine du poulet. Ces deux mesures étaient prises à l'aide d'un micromètre donnant le vingtième de millimètre et muni de deux mâchoires terminées par un disque plat de I centimètre de diamètre (fig. I).

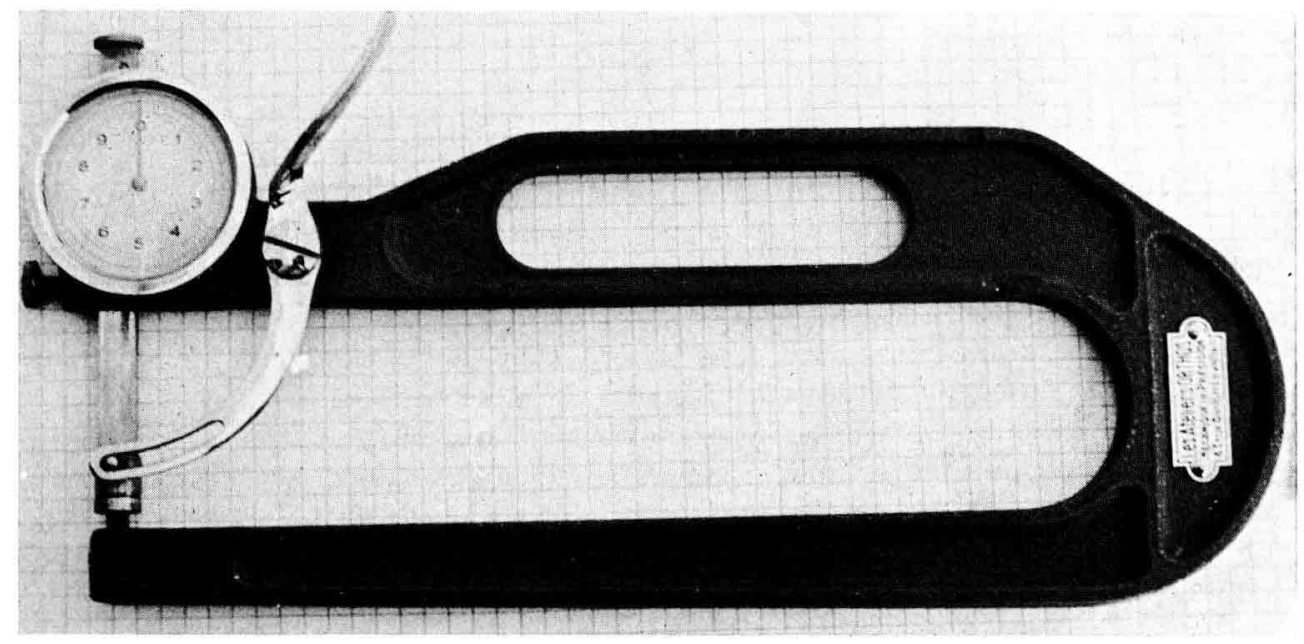

FIG. I. - Vue du micromìtre utilisé pour mesurer les épaisseurs de peau

Les carrés du support ont $5 \mathrm{~mm}$ de côté 
3. Épaisseur de la peau de la patte, estimée de deux manières différentes :

a) mesure directe, au micromètre, d'un fragment de peau prélevé au milieu et dans la partie arrière du tarso-métatarse ;

b) estimation indirecte obtenue en mesurant le petit diamètre de la patte environ au milieu du tarso-métatarse et le diamètre de l'os au même endroit. La mesure du petit diamètre permet d'éviter le groupe de tendons situés à la partie postérieure du tarso-métatarse. La différence des deux dia. mètres représente deux fois l'épaisseur de la peau plus le tissu conjonctif situé entre la peau et l'os, mais qui est ici peu important. Les diamètres étaient mesurés au dixième de millimètre près, à l'aide d'un pied à coulisse.

Sur la carcasse éviscérée, nous avons disséqué et pesé, séparément à droite et à gauche, la peau des deux premiers segments de l'aile, la peau de l'ensemble cuisse + pilon et la peau du reste de la carcasse. L'ensemble des ces trois éléments est considéré comme représentant la totalité de la peau de la carcasse. Dans la mesure du possible, nous avons évité d'inclure dans la peau les dépôts graisseux sous-cutanés et les petits muscles peauciers. La peau des deux pattes a également été disséquée et pesée.

\section{Traitement statistique des données}

Nous avons d'abord cherché à estimer la répétabilité des mesures prises. Dans le cas des mesures d'épaisseur nous avons trois répétitions à droite et trois répétitions à gauche, ce qui nous a permis de faire une analyse de variance selon le modèle suivant (expérience factorielle à deux niveaux, modèle II de SNEDECOR, 1956) :

\begin{tabular}{|c|c|c|}
\hline Source de variation & Degrés de liberté & $\begin{array}{l}\text { Composition } \\
\text { du carré moyen }\end{array}$ \\
\hline $\begin{array}{l}\text { Totale } \ldots \ldots \ldots \ldots \ldots \\
\text { Poulets } \ldots \ldots \ldots \ldots \ldots \ldots \\
\text { Côtés } \ldots \ldots \ldots \ldots \ldots \ldots \\
\text { Interaction... } \\
\text { Résiduelle } \ldots \ldots \ldots \ldots \ldots \ldots\end{array}$ & $\begin{array}{r}149 \\
2 / 4 \\
1 \\
24 \\
100\end{array}$ & $\begin{array}{l}\sigma_{e}^{2}+3 \sigma_{a b}^{2}+6 \sigma^{2} a \\
\sigma_{e}^{2}+3 \sigma_{a b}^{2}+75 \sigma_{b}^{2} b \\
\sigma_{e}^{2}+3 \sigma_{a b}^{2} \\
\sigma_{e}^{2}\end{array}$ \\
\hline
\end{tabular}

où $\sigma_{a}^{2}$ représente la variance "poulet ", $\sigma_{b}^{2}$ la variance " côté ", $\sigma_{a b}^{2}$ la variance " interaction " et $\sigma_{e}^{2}$ la la variance résiduelle, c'est-à-dire celle correspondant aux erreurs de mesure. Nous avons considéré que la répétabilité de la mesure était donnée par le coefficient de corrélation intra-classe pour un même côté, c'est-à-dire :

$$
\rho=\frac{\sigma_{a}^{2}}{\sigma_{u}^{2}+\sigma_{\ell}^{2}}
$$

Dans le cas des pesées de peau, nous n'avons qu'une mesure à droite et une mesure à gauche. L'analyse de variance se résume de la façon suivante:

\begin{tabular}{|c|c|c|}
\hline Source de variation & Degrés de liberté & $\begin{array}{l}\text { Composition } \\
\text { du carré moyen }\end{array}$ \\
\hline Totale. & 49 & \\
\hline Poulet $\ldots \ldots \ldots \ldots \ldots$ & 24 & $\sigma_{e}^{2}+2 \sigma_{a}^{2}$ \\
\hline Résiduelle......... & 25 & $\sigma_{e}^{2}$ \\
\hline
\end{tabular}

et le coefficient de corrélation intra-classe devient un coefficient de corrélation droite-gauche. Nous avons également calculé la corrélation droite-gauche pour les mesures d'épaisseur, à partir de la somme des trois répétitions de chaque côté. 
Nous avons ensuite recherché si les variables étudiées permettaient de prédire l'importance de la peau sur la carcasse entière, en calculant les corrélations totales avec le poids de cette peau. Pour tenir compte de l'influence du poids du poulet et de son état d'engraissement, nous avons également calculé les corrélations partielles à poids de carcasse constant, les corrélations partielles à engraisse. ment (c'est-à-dire, ici, la racine carrée du poids du dépôt gras abdominal) constant, ainsi que les corrélations partielles à poids de carcasse et engraissement constants.

\section{RÉSULTATS ETT DISCUSSION}

Dans le tableau I sont indiqués les paramètres statistiques des variables étudiées et les coefficients de répétabilité.

Pour les pesées de peau, l'unité de mesure est faible vis-à-vis de l'écart-type. La corrélation droite-gauche est élevée (de l'ordre de 0,9 ), ce qui permet de supposer que les erreurs de dissection sont faibles. On pourrait se contenter de ne disséquer la peau que sur un seul des deux membres.

Dans le cas des mesures d'épaisseur, les det1x coefficients calculés sont du même ordre de grandeur. Il semble donc que les erreurs dues à une dissymétrie possible des carcasses soient du même ordre de grandeur que les erreurs de mesure. Les valeurs obtenues sont satisfaisantes pour la mesure directe de l'épaisseur de la peau de la patte. Nous considérons qu'elles sont faibles dans les trois autres cas. Une première explication pourrait venir du fait que l'unité de mesure est grande vis-à-vis de l'écarttype (40 à $80 \mathrm{p}$. Ioo). Cependant, la répétabilité est bonne pour la mesure directe de l'épaisseur de la peau de la patte. Il y a donc, en plus, une imprécision inhérente à la mesure des trois autres épaisseurs. Pour la mesure au niveau de l'aile et de la cuisse, on peut penser à une pression irrégulière des mâchoires du micromètre, ou aux irrégularités de la surface de la peau due à la présence d'un nombre plus ou moins grand de bulbes plumaires. On améliorerait certainement les résultats en utilisant un appareil de mesure plus précis, donnant par exemple le centième de millimètre, et exerçant une pression faible et constante sur la peau. Pour l'estimation indirecte faite au niveau de la patte, on retrouve l'imprécision qui s'attache aux mesures par différence, même si les deux membres de la différence sont connus avec précision : dans le cas présent, le coefficient de corrélation intra-classe était de 0,97 pour le diamètre de la patte entière et de 0,98 pour le diamètre de l'os.

Le tableau 2 donne les corrélations calculées entre, d'une part, le poids de peau de la carcasse entière, et, d'autre part, le poids de la carcasse, la racine carrée du poids de la graisse abdominale, ainsi que chacune des variables figurant au tableau $\mathrm{I}$.

Le poids total de la peau est assez fortement lié au poids de la carcasse $(r=0,8)$, ce qui confirme les résultats de MCNALLY et KRAFT (I958) qui ont trouvé une corrélation de 0,93. Sur des poulets Leghorn abattus à différents âges, LATIMER (I924) observait de son côté une forte liaison entre le poids vif vide et le poids de la peaul. Dans notre échantillon, le poids total de peau n'est pas très fortement lié à l'état d'engraissement : la corrélation totale, comme la corrélation partielle à poids constant, est de l'ordre de 0,4. McNaILY et Kraft (1958) indiquent, sans donner de chiffre, que le poids de la peau est fortement lié au poids de la graisse. Essary et al. (I96I) trouvent une corrélation de 0,62 entre le pourcentage de peau de la carcasse et le pourcentage de graisse abdominale, valeur qu'il faut comparer à notre corréla- 


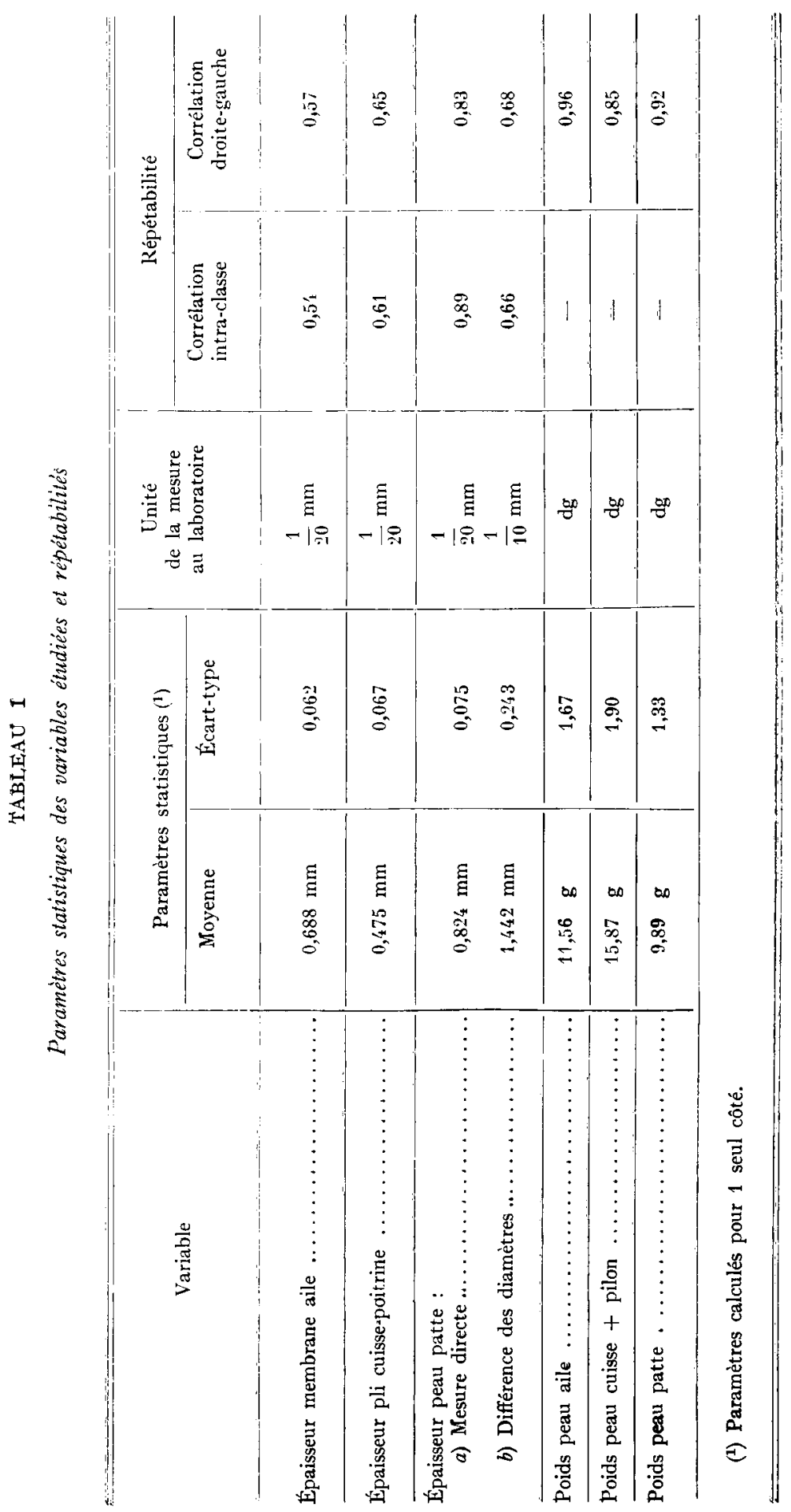




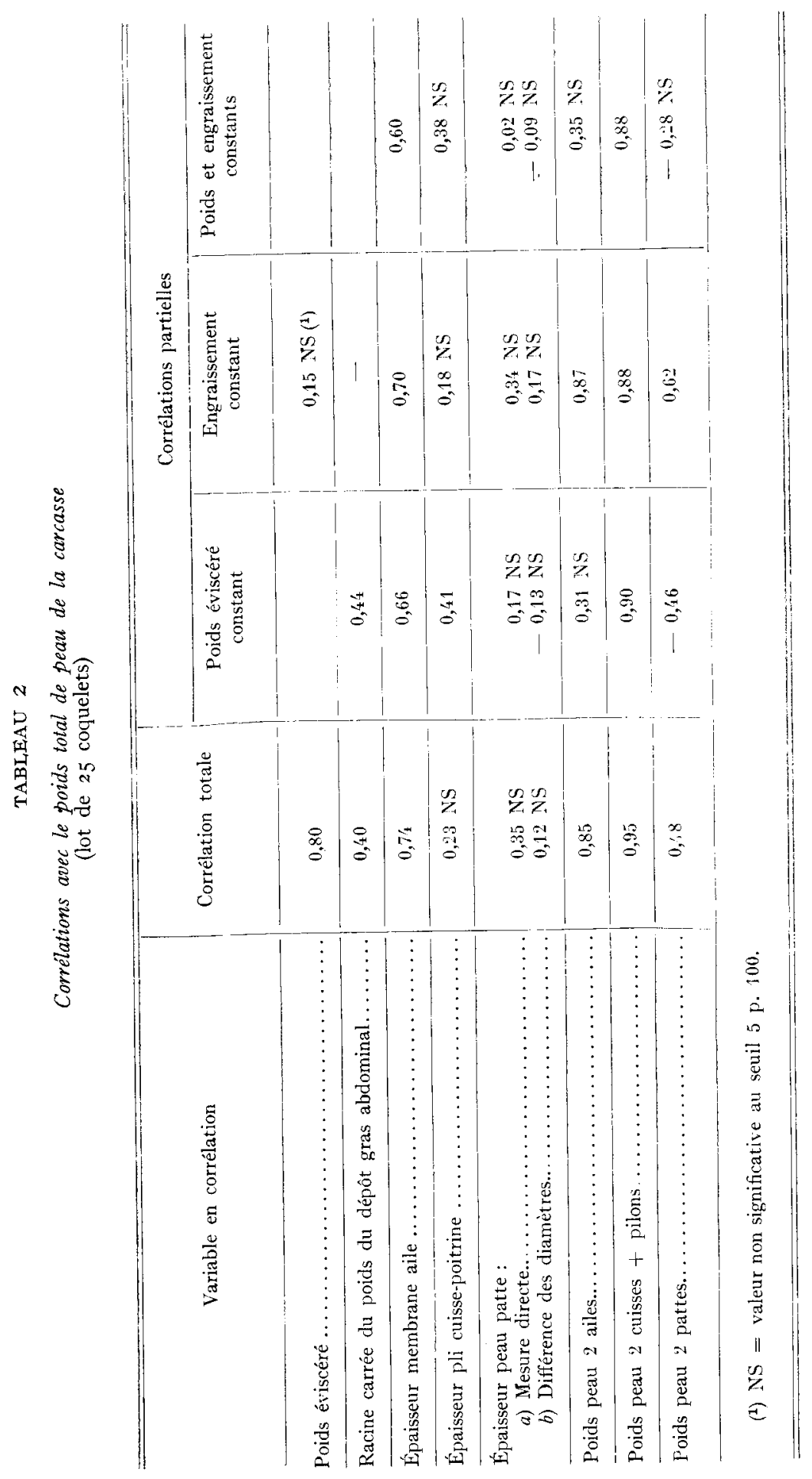


tion partielle à poids constant et qui est nettement plus élevée. Il est possible que cette différence soit due à la souche ou à notre technique expérimentale. En effet, sur des données plus nombreuses (les 315 coquelets étudiés dans RICARD et ROUviER, I967, qui provenaient de la même souche mais étaient abattus à I I semaines), nous avons trouvé une corrélation totale de 0,47 et une corrélation partielle à poids constant de 0,34 , chiffres peu différents de ceux que nous obtenons ici.

Le tableau 2 montre que, parmi les mesures d'épaisseur, seule l'épaisseur de la peau de l'aile présente des corrélations significatives avec le poids total de peau de la carcasse. On peut se demander si ces mesures répondent à ce qu'on attend d'elles, à savoir l'estimation de la "finesse " de la peau. Nous n'avons pas étudié les facteurs de variation de l'épaisseur de la peau sur toute la carcasse, mais nous avons pris des mesures en des endroits où il n'y a pratiquement pas de graisse sous-cutanée. L'épaisseur du pli poitrine-cuisse et celle de la peau de la patte semblent varier de façon indépendante du poids total de la peau. On peut alors penser qu'il s'agit bien d'une mesure intrinsèque de la finesse de la peau. MIais cette hypothèse demande à être confirmée sur un plus grand nombre de données.

Le poids de peau de l'ensemble cuisses + pilons est la variable qui représente le mieux la quantité totale de peau de la carcasse : la corrélation totale est très forte $(r=0,95)$, de même que les trois corrélations partielles calculées $(r$ de l'orđre de 0,9$)$; c'est donc le caractère qu'il faut mesurer si on veut estimer rapidement l'importance de la peau chez le Poulet. Dans le cas présent, l'équation de régression s'écrit :

$$
y=3,40 x-2,07
$$

où $x$ est le poids de peau des deux cuisses et des deux pilons et $y$ le poids total de peau de la carcasse, $x$ et $y$ étant exprimés en grammes. Nous avons vu qu'il suffisait de ne disséquer qu'un seul des deux membres. Dans la pratique, on pourra calculer le pourcentage du poids de peau d'une cuisse et d'un pilon par rapport au poids total de cette cuisse et de ce pilon. Dans l'échantillon étudié ici, ce pourcentage présente une corrélation de 0,89 avec le rapport du poids total de peau au poids de la carcasse éviscérée.

A priori, on pouvait penser utiliser des mesures prises au niveau de la patte pour ne pas avoir à disséquer la carcasse proprement dite. Les résultats du tableau 2

\section{TABLEAU 3}

Corrélations avec le poids total de peau de la carcasse (lot complémentaire de 20 corquelets)

\begin{tabular}{|c|c|c|c|c|}
\hline & & \multicolumn{3}{|c|}{ Corrélations partielles } \\
\hline Variable en corrélation & $\begin{array}{l}\text { Corrélation } \\
\text { totale }\end{array}$ & $\begin{array}{l}\text { Poids éviscéré } \\
\text { constant }\end{array}$ & $\begin{array}{l}\text { Engraissement } \\
\text { constant }\end{array}$ & $\begin{array}{l}\text { Poids et } \\
\text { engraissement } \\
\text { constants }\end{array}$ \\
\hline $\begin{array}{l}\text { Epaisseur peau patte : } \\
\text { b) Différence des diamètres }\end{array}$ & $0,23 \mathrm{NS}$ & $0,0 \prime \mathrm{NS}$ & $-0,07 \mathrm{NS}$ & $0,05 \mathrm{NS}$ \\
\hline Poids peau 2 pattes .... & 0,67 & $-0,09 \mathrm{NS}$ & 0,70 & $0,0_{ \pm}^{\prime} \mathrm{NS}$ \\
\hline
\end{tabular}


montrent que ce n'est pas possible, au moins en ce qui concerne l'estimation de l'importance de la peau. On observe même des corrélations partielles négatives pour le poids de peau des pattes et l'épaisseur estimée par la différence des diamètres. Nous avons vérifié ce dernier point sur un autre lot de 20 poulets de la même souche et abattus au même âge. Les résultats sont indiqués dans le tableau 3 : les valeurs obtenues sont du même ordre de grandeur que celles du tableau 2 . Il se confirme donc que les mesures prises au niveau de la patte ne permettent pas d'estimer correctement l'importance de la peau chez le poulet.

Reçu pour publication en oclobre $\mathrm{s} 968$.

\section{REMERCIEMENTS}

Nous remercions MM. P. DeLpech et R. Rouvier pour les suggestions qu'ils nous ont fajte: après avoir lu notre manuscrit.

\section{SUMMARY}

ESTIMATION OF THICKNESS AND WEIGHT OF CHICKEN SKIN

Skin thickness was estimated on wing, shank and skin fold between breast and thigh of 25 chickens. The skin of wing, thigh + leg and shank was flayed and weighed.

Total bodyskin weight is highly correlated with carcass weight : $r=0.8$, but the correlation with fat weight is much lower : $r=0.4$.

Thickness estimations are not accurate, except for the direct estimation of shank skin thickness. More data are required before concluding whether our measurements allow an estimation of skin thickness irrespective of bodyskin weight and fat. Correlations between thickness and total bodyskin weight are not significant, except for wing.

Thigh + leg skin weight $(x)$ appears to allow the best estimation of total bodyskin weight $(y)$. The correlation is 0.95 and the regression equation is :

( $x$ and $y$ in grams).

$$
y=3.40 x-2.07
$$

\section{RÉFÉRENCES BIBLIOGRAPHIQUES}

Adnms A. W., Carlson C. W., Kahrs A. J., FRY J. L., 1967. Relationships of several environmental factors to the incidence of breast blisters in large type male market turkeys. Poult. Sci., 46, 569-577.

Delpech P., Ricard F.-H., 1965. Relation entre les dépôts adipeux viscéraux et les lipides corporels chez le Poulet. Ann. Zootech., 14, I8I-189.

Dolecek R. L., Wilson W. D., Poley W. E., I94I. Physical measurements of carcass quality in roasters. Poult. Sci., 20, I6r-I70.

Essary E. O., Dawson L. E., Wisman E. L., Holmes C. E., ig6r. Influence of fat and protein in broiler rations on fat deposition. Poult. Sci., 40, 1399 (Abstract).

LAtimer H. B., I924. Postnatal growth of the body, systems, and organs of the single-comb White Leghorn chicken. J. Agric. Res., 29, 363-397.

McNally E. H., KRafT A. A., 1958. The body skin of market poultry. Poull. Sci., 37, i 226 (Abstract).

RICARd F.-H., Rouvier R., I967. Étude de la composition anatomique du poulet. I. Variabilité de la répartition des différentes parties corporelles chez des coquelets Bresse-pile. Ann. Zootech. 16, 23-39.

Snedecor G. W., I956. Statistical methods, and Edition. The Iowa State College Press, Ames. 